\title{
Fixed Switching Period Discrete-Time Sliding Mode Current Control of a PMSM
}

\author{
Víctor Repecho, Domingo Biel and Antoni Arias
}

\begin{abstract}
A fixed switching period sliding mode control (SMC) for Permanent Magnet Synchronous Machines (PMSMs) is presented. The aim of the paper is to design a SMC that improves the traditional PI based Field Oriented Control (FOC) transient response, as well as to reduce the switching frequency variations of the Direct Torque Control (DTC). Such SMC requires a decoupling method of the control actions, which also brings constant switching functions slopes. These constant slopes allow to calculate the required hysteresis band value to control the switching frequency. The digital implementation degrades the performance of the hysteresis comparator and as a consequence, the previously calculated band becomes inaccurate to regulate the switching frequency. In order to recover the analogue hysteresis band comparator performance, a predictive algorithm is proposed. Finally, a set of experimental results with constant switching frequency during a torque reversal and speed control tests are provided.
\end{abstract}

Index Terms-PMSM, sliding mode control, adaptive hysteresis comparator, fixed switching frequency

\section{INTRODUCTION}

$\mathbf{P}$ ERMANENT Magnet Synchronous Machines (PMSMs) have gained market due to their inherent advantages such as higher efficiency, lower inertia and lower volume when compared to other AC machines [1]. Nowadays, they have become a widely used electrical machine not only for traditional or general purpose applications but also for the state of the art ones such as wind turbines and electric vehicles [2]-[4]. Field Oriented Control (FOC) [5] and Direct Torque Control (DTC) [6], [7] are the most widely used high performance control strategies for controlling AC Machines including the PMSM ones [8]. Among other issues, it could be established that the most important advantage of the FOC is its constant switching frequency while for the DTC is its high dynamics.

In the case of the DTC, the main drawbacks are the variable switching frequency, the high torque and current ripples at low speeds [9], while for FOC schemes the high sensibility of the controller performance to the motor parameters entails the main challenge [10]. Several publications can be found in the literature where these drawbacks have been mitigated for DTC in [11]-[16]. Related to FOC schemes, the researches have

Manuscript received Month xx, 2xxx; revised Month xx, xxxx; accepted Month $\mathrm{x}, \mathrm{xxxx}$. This work was supported in part by the spanish projects DPI2013-41224-P (Ministerio de Educación) and 2014 SGR 267 (AGAUR). V.Repecho, D.Biel and A.Arias are with the Institute of Industrial and Control Engineering, Universitat Politècnica de Catalunya, 08028, Barcelona, Catalonia, Spain. (e-mails: victor.repecho.del@upc.edu, domingo.biel@upc.edu, antoni.arias@upc.edu). been focused in estimating the motor parameters in order to improve the system robustness. A summary of the estimation and observation techniques is presented in [17].

Two of the main benefits of the Sliding Mode Control (SMC) [18] are its high dynamic response during transients and its robustness. In this sense, SMC performance is comparable to the DTC one. Even though in both schemes the switching frequency is inherently variable, the SMC provides a set of mathematical tools which allows to fix the switching frequency, achieving also one of the main advantages of FOC schemes. Accordingly, SMC is an alternative to the popular FOC and DTC. In [18] sliding mode controllers together with sliding mode observers are designed for PMSM current and speed regulation. Another application of sliding mode observer can be found in [19], [20] for PMSM sensorless operation. Unfortunately, the design of a first order SMC in the $d q$ plane yields a control action that leads to a continuous set of values in $a b c$ and it cannot be implemented even in a continuous time approximation of the Voltage Source Inverter (VSI). This fact, together with discretization of the control laws have been tackled in several works for the SMC application. In [21] a discrete-time quasi sliding mode control is designed in $d q$ frame, in order to control the motor speed. The provided $d q$ voltages are transformed to the $a b c$ frame and directly implemented by means of a Pulse Width Modulator (PWM) to the inverter at fixed frequency. The control action includes a discontinuous term that ensures sliding motion only outside of a given sector. Inside this sector, the control provides a quasi sliding motion which requires the knowledge of the system parameters.

Similarly, in [22] the $d q$ controllers are implemented by means of a discrete-time sliding mode introduced by Gao in [23]. Again, this technique requires the knowledge of the motor parameters for the control action computation. In [24] an SMC in the $a b c$ currents is proposed and the control laws are directly deduced through a Look up Table (LUT). The use of the LUT provokes a variable switching frequency of the control actions, which can be understood as a drawback for this application. Indeed, the switching frequency of the control actions is a well-known problem to be tackled when an SMC has to be implemented. Hysteresis comparators are usually employed to implement SMC. They can be easily built using analogue circuitry [25], [26] but their implementation using micro-controllers degrades the performance due to the sampling period.

The main contribution of this research is the design of an SMC for PMSMs keeping the advantages of DTC and 
FOC, which are high dynamics and constant switching frequency, respectively. Instead of designing the SMC in the $d q$ coordinates, it is proposed to define the switching surfaces in the $a b c$, which delivers the control laws directly for the VSI. A decoupling technique, which allows to find a set of decoupled sliding surfaces [18], has been used similarly to the procedure proposed in [27] for induction machines. Such decoupling technique simplifies the implementation of the proposed SMC by a microcontroller. On the one hand, the decoupling technique allows the use of a simple but effective prediction algorithm, similarly to the ones proposed in [28][31], that digitally emulates the performance of a continuous time hysteresis comparator, eliminating the sampling and the computing delays effects. On the other hand, once the continuous time hysteresis comparator performance is recovered, the well-known problem of the variable switching frequency of the SMC is mitigated using a variable hysteresis band technique. These methodologies, of emulating the hysteresis comparators and using a time varying band to regulate the switching frequency constitute a useful tool to digitally implement the $\mathrm{SMC}$ in power converters.

Finally, the proposed controllers together with aforementioned implementation methodologies are experimentally tested in an off-the-shelf PMSM with satisfactory results.

\section{SLIDING Mode CuRRENT CONTROL OF PMSMS}

\section{A. PMSM model}

The PMSM electrical model equations in the stationary reference frame $(a b c)$ [1] are given by:

$$
\mathbf{L} \frac{d \mathbf{i}}{d t}=\mathbf{v}_{\mathbf{x n}}-R \mathbf{i}-\mathbf{e}\left(\omega_{e}, \theta_{e}\right)
$$

where $\mathbf{i}=\left[i_{a}, i_{b}, i_{c}\right]^{T}, \mathbf{v}_{\mathbf{x n}}=\left[v_{a}-v_{n}, v_{b}-v_{n}, v_{c}-v_{n}\right]^{T}$, $\mathbf{e}\left(\omega_{e}, \theta_{e}\right)=\left[e_{a}, e_{b}, e_{c}\right]^{T}$ are the stator currents, stator voltages and back-emf voltages, respectively. The back-emf voltages are given by:

$$
\begin{gathered}
e_{a}=-\Psi_{M} \omega_{e} \sin \theta_{e} \\
e_{b}=-\Psi_{M} \omega_{e} \sin \left(\theta_{e}+2 \pi / 3\right) \\
e_{c}=-\Psi_{M} \omega_{e} \sin \left(\theta_{e}-2 \pi / 3\right)
\end{gathered}
$$

being $\Psi_{M}$ the rotor permanent flux, $\omega_{e}$ the electrical motor speed and $\theta_{e}$ the electrical motor angle.

The matrix $\mathbf{L}$ is defined as follows.

$$
\mathbf{L}=\left[\begin{array}{ccc}
L_{a} & 0 & 0 \\
0 & L_{b} & 0 \\
0 & 0 & L_{c}
\end{array}\right]
$$

where $L_{a}, L_{b}$ and $L_{c}$ are the stator inductances.

The right side of the figure 1 shows the common structure for the motor drive. The VSI is able to perform the controlled voltages at the points $a, b, c$, through the complementary operation of the switches. Specifically the voltages $v_{a}, v_{b}, v_{c}$ can be connected only to $v_{b u s}$ or $-v_{b u s}$ through the control signals $u_{a}, u_{b}, u_{c}$ as:

$$
\mathbf{v}=v_{\text {bus }} \mathbf{u}
$$

defining $\mathbf{u}=\left[u_{a}, u_{b}, u_{c}\right]^{T}$ and $\mathbf{v}=\left[v_{a}, v_{b}, v_{c}\right]^{T}$. The control signals $u_{a}, u_{b}, u_{c}$, which control the state of the VSI switches, just can take the discrete values 1 or -1 . Finding the voltage $v_{n}$, is possible to relate (1) with the VSI control signals $u_{a}, u_{b}, u_{c}$. From the structure shown in Figure 1, the voltage $v_{n}$ can be found assuming the circuit linear and applying the well-known superposition principle.

$$
\begin{gathered}
v_{n}=\frac{L_{b} L_{c}\left(v_{b u s} u_{a}-e_{a}\right)}{L_{T}}+\frac{L_{a} L_{c}\left(v_{b u s} u_{b}-e_{b}\right)}{L_{T}} \\
+\frac{L_{a} L_{b}\left(v_{b u s} u_{c}-e_{c}\right)}{L_{T}}
\end{gathered}
$$

where $L_{T}=L_{b} L_{c}+L_{a} L_{c}+L_{a} L_{b}$. The expression can be further simplified taking into account that in a balanced system the relation $e_{a}+e_{b}+e_{c}=0$ holds, and the difference among the stator inductance will be small, yielding:

$$
v_{n} \approx \frac{v_{\text {bus }}}{L_{T}}\left[u_{a} L_{b} L_{c}+u_{b} L_{a} L_{c}+u_{c} L_{a} L_{b}\right]
$$

Remark 1. Notice that the voltage $v_{n}$ defined in (5) results in the expression

$$
v_{n}=\frac{v_{b u s}}{3}\left[u_{a}+u_{b}+u_{c}\right]
$$

if all the inductance values are considered equal. Expression (5) is useful when the inductance values are different but close among them.

Merging (1), (4) and (5), the model, which includes the control signals of the VSI in the stator currents dynamics, is rewritten in (6).

$$
\mathbf{L} \frac{d \mathbf{i}}{d t}=-R \mathbf{i}-\mathbf{e}\left(\omega_{e}, \theta_{e}\right)+v_{b u s} \mathbf{L B} \mathbf{u}
$$

where

$$
\mathbf{B}=\frac{1}{L_{T}}\left[\begin{array}{ccc}
L_{b}+L_{c} & -L_{c} & -L_{b} \\
-L_{c} & L_{a}+L_{c} & -L_{a} \\
-L_{b} & -L_{a} & L_{a}+L_{b}
\end{array}\right]
$$

The term $\mathbf{e}\left(\omega_{e}, \theta_{e}\right)$ in (6) can be considered as an external unknown signal from the motor current dynamics point of view, since it depends on the motor speed and rotor angle.

\section{B. Decoupled Sliding Mode PMSM Current Control}

The control objective is to design three switching surfaces such that the motor currents in the $a b c$ frame track the desired values. The star connection of the motor windings entails the restriction $i_{a}+i_{b}+i_{c}=0$. Therefore, the three PMSM currents will be controlled by just two control signals and the third control signal is being used to regulate the average value of the discontinuous voltage $v_{n}$, whose switching surface is defined in (8).

$$
\mathbf{S}=\left[i_{a}^{*}-i_{a}, i_{b}^{*}-i_{b}, \int\left(v_{n}^{*}-v_{n}\right) d t\right]^{T}
$$

where the superscript $*$ stands for the reference value.

The proposed decoupled sliding mode PMSM current control leads to a high dynamics tracking. When the system state is outside the sliding surface, the system trajectories will reach $\mathbf{S}=0$ after a brief transient. Once the system is on sliding motion, $i_{a}^{*}, i_{b}^{*}$ will be perfectly tracked by the currents $i_{a}, i_{b}$, 


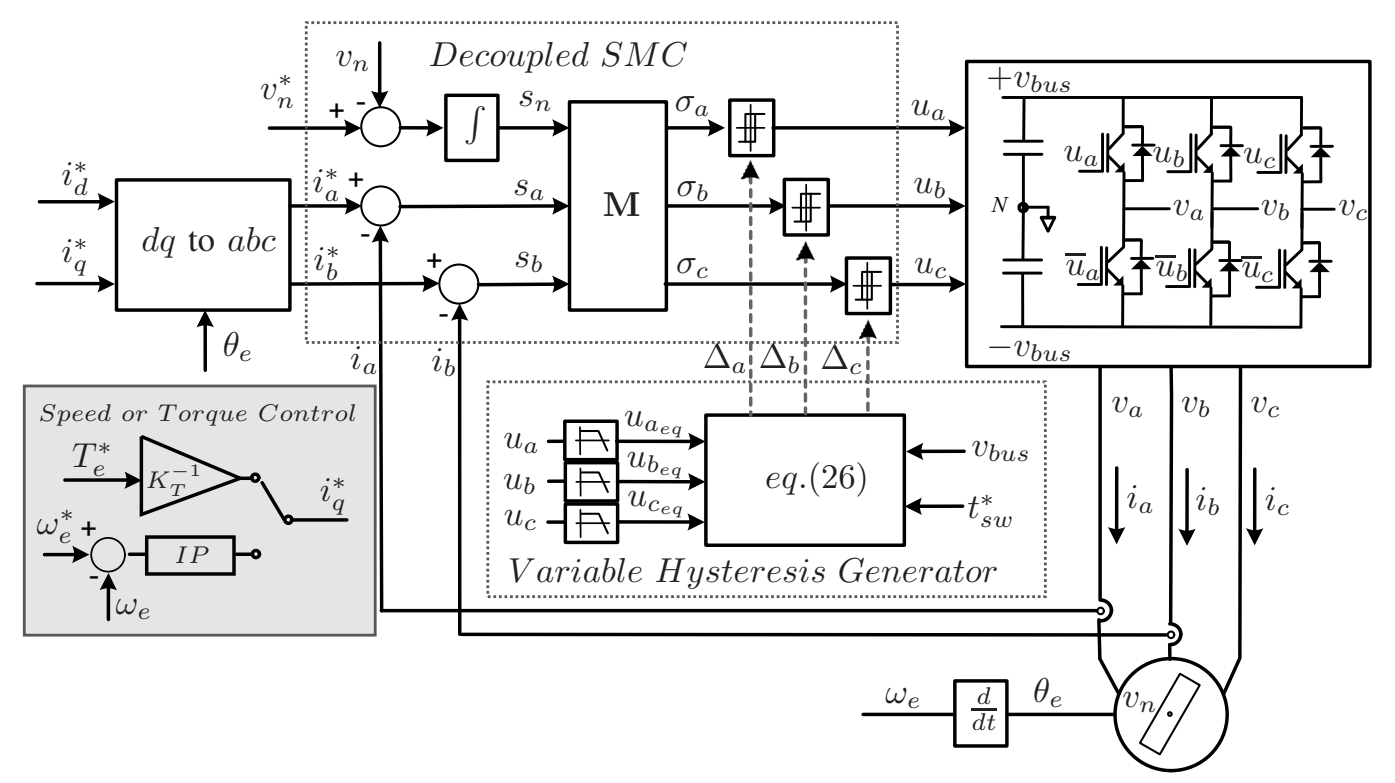

Fig. 1. Diagram of the proposed PMSM drive, where the SMC decoupling control and the variable hysteresis band generator are highlighted.

despite of the unknown time varying signal $e\left(\omega_{e}, \theta_{e}\right)$. Notice that such perfect tracking of the reference is just achieved in the ideal case, which assumes infinite switching frequency. The real sliding motion is described in Section III.

Applying time derivative to (8) and replacing (5) and (6), the sliding mode equation becomes:

$$
\dot{\mathbf{S}}=\mathbf{f}_{\mathbf{1}}-\frac{v_{b u s}}{L_{T}} \mathbf{C} \mathbf{u}
$$

where

$\mathbf{C}=\left[\begin{array}{ccc}L_{b}+L_{c} & -L_{c} & -L_{b} \\ -L_{c} & L_{a}+L_{c} & -L_{a} \\ L_{b} L_{c} & L_{a} L_{c} & L_{a} L_{b}\end{array}\right] ; \mathbf{f}_{\mathbf{1}}=\left[\begin{array}{c}\dot{i}_{a}^{*}+\frac{R}{L_{a}} i_{a}+\frac{e_{a}}{L_{a}} \\ \dot{i}_{b}^{*}+\frac{R}{L_{b}} i_{b}+\frac{e_{b}}{L_{b}} \\ v_{n}^{*}\end{array}\right]$

Note how each surface time derivative depends on all the control signals. Finite time convergence of $\mathbf{S}=0$ is possible by means of a Lyapunov function candidate $V=0.5 \mathbf{S}^{T} \mathbf{S}$, and designing a control such that $\dot{V}<0$ [18], nevertheless this task is not easy. Moreover, the behaviour of the switching surfaces inside the hysteresis band is complicated to analyse, and hinders the task to control the effective switching frequency.

This problem is overcome by applying a well-known decoupling control procedure ( [18], [27], [32]) that allows to find a new set of switching surfaces which could be independently controlled by each control signal. The new set of switching surfaces are defined in (10).

$$
\sigma=\mathrm{MS}
$$

where $\mathbf{M}$ is a non-singular decoupling matrix and $\boldsymbol{\sigma}=$ $\left[\sigma_{a}, \sigma_{b}, \sigma_{c}\right]^{T}$ is the new surfaces set.

The sliding mode equation of the new surfaces set is:

$$
\dot{\boldsymbol{\sigma}}=\mathbf{M f}_{\mathbf{1}}-\frac{v_{\text {bus }}}{L_{T}} \mathbf{M C} \mathbf{u}
$$

The decoupling matrix, $\mathbf{M}$, has to be defined such that the matrix MC becomes diagonal. Notice how the requirement for matrix $\mathbf{C}$ is to be non-singular, in that way $\mathbf{M}$ exists.
Such property for $\mathbf{C}$ is achieved through a well designed set of switching surfaces in (8). Specifically, the determinant for matrix $\mathbf{C}$ becomes $\operatorname{det}(\mathbf{C})=L_{T}^{2}$. Otherwise, the decoupling process cannot be performed. Therefore, a reasonable choice for $M$ is:

$$
\mathbf{M}=L_{T} \mathbf{C}^{-1}=\left[\begin{array}{ccc}
L_{a} & 0 & 1 \\
0 & L_{b} & 1 \\
-L_{c} & -L_{c} & 1
\end{array}\right]
$$

According to the definition of $\mathbf{M}$, the sliding mode equation becomes:

$$
\dot{\boldsymbol{\sigma}}=\mathbf{f}_{\mathbf{2}}-v_{\text {bus }} \mathbf{I} \mathbf{u}
$$

where $\mathbf{I}$ is the $3 \times 3$ identity matrix and

$$
\mathbf{f}_{\mathbf{2}}=\left[\begin{array}{l}
f_{2_{a}} \\
f_{2_{b}} \\
f_{2_{c}}
\end{array}\right]=\left[\begin{array}{c}
L_{a} \dot{i}_{a}^{*}+R i_{a}+e_{a}+v_{n}^{*} \\
L_{b} \dot{i}_{b}^{*}+R i_{b}+e_{b}+v_{n}^{*} \\
-L_{c}\left[\dot{i}_{a}^{*}+\frac{R i_{a}+e_{a}}{L_{a}}+\dot{i}_{b}^{*}+\frac{R i_{b}+e_{b}}{L_{b}}\right]+v_{n}^{*}
\end{array}\right]
$$

Remark 2. The surface set based on the current errors: $\mathbf{S}=\left[i_{a}^{*}-i_{a}, i_{b}^{*}-i_{b}, i_{c}^{*}-i_{c}\right]$ produces a singular matrix for $\mathrm{C}$, which hinders the decoupled method application. This analysis has been omitted for the sake of brevity.

The control law proposed in Theorem 1 ensures that the system trajectories converge in finite time to the manifold $\sigma=$ 0 and therefore, by definition of $\mathbf{M}$, to $\mathbf{S}=0$.

Theorem 1. Assume that the initial switching surfaces shown in (8) are decoupled using the non-singular matrix $\mathbf{M}$ (12), providing the set of switching surfaces shown in (10). Let also assume that $\mathbf{f}_{2}$ is bounded and being such bound known and equal to $\mathbf{f}_{\mathbf{2}_{\mathrm{M}}}=\left[f_{2_{a} M}, f_{2_{b} M}, f_{2_{c} M}\right]^{T}$, where $f_{2_{i} M}=$ $\left|f_{2_{i}}\right|_{\text {MAX }}$ for $i \in a, b, c$. At these conditions, with the control law

$$
\mathbf{u}=\operatorname{sign}(\boldsymbol{\sigma})
$$


the current values of $i_{a}, i_{b}$ and the mean value of $v_{n}$ converge asymptotically to their reference values $i_{a}^{*}, i_{b}^{*}, v_{n}^{*}$ in finite time if the bus voltage $v_{\text {bus }}$ fulfils the inequality $v_{b u s}>\max \left\{f_{2_{a} M}\right.$, $\left.f_{2_{b} M}, f_{2_{c} M}\right\}+\eta$, being $\eta>0$.

Proof. Selecting the Lyapunov function candidate

$$
V=0.5 \boldsymbol{\sigma}^{T} \boldsymbol{\sigma}
$$

the time derivative of $V$ yields

$$
\dot{V}=\boldsymbol{\sigma}^{T} \mathbf{f}_{\mathbf{2}}-v_{\text {bus }} \boldsymbol{\sigma}^{T} \mathbf{u}
$$

which is equivalent to

$$
\dot{V}=\sigma_{a} f_{2_{a}}+\sigma_{b} f_{2_{b}}+\sigma_{c} f_{2_{c}}-v_{b u s}\left[\sigma_{a} u_{a}+\sigma_{b} u_{b}+\sigma_{c} u_{c}\right]
$$

using the control law defined in (15) and, taking into account that the values $f_{2_{a}}, f_{2_{b}}, f_{2_{c}}$ are bounded by $f_{2_{a} M}, f_{2_{b} M}, f_{2_{c} M}$, one gets

$\dot{V} \leq\left|\sigma_{a}\right| f_{2_{a} M}+\left|\sigma_{b}\right| f_{2_{b} M}+\left|\sigma_{c}\right| f_{2_{c} M}-v_{b u s}\left[\left|\sigma_{a}\right|+\left|\sigma_{b}\right|+\left|\sigma_{c}\right|\right]$,

which can be rewritten as

$\dot{V} \leq\left|\sigma_{a}\right|\left[f_{2_{a} M}-v_{b u s}\right]+\left|\sigma_{b}\right|\left[f_{2_{b} M}-v_{b u s}\right]+\left|\sigma_{c}\right|\left[f_{2_{c} M}-v_{b u s}\right]$

Hence $\dot{V}<-\eta\left[\left|\sigma_{a}\right|+\left|\sigma_{b}\right|+\left|\sigma_{c}\right|\right]$ holds for the selected value of $v_{b u s}$ of Theorem 1 which satisfies $v_{b u s}>\max \left\{f_{2_{a} M}\right.$, $\left.f_{2_{b} M}, f_{2_{c} M}\right\}+\eta$, being $\eta>0$. This condition implies that the functions $\sigma_{a}, \sigma_{b}, \sigma_{c}$ will converge to the manifold $\sigma=0$ in finite time. Therefore, due to the non-singularity of $\mathbf{M}$, and recalling (10), the perfect tracking is reached in finite time as well.

Remark 3. Since $\dot{i}_{a}^{*}, \dot{i}_{b}^{*}$ appear on $\mathbf{f}_{\mathbf{2}}$, it is likely that after a current transient, the previous condition does not hold and $i_{a} \neq i_{a}^{*}, i_{b} \neq i_{b}^{*}, v_{n} \neq v_{n}^{*}$. However, once the transient disappears, the theorem condition will hold again and the real values will converge in finite time to the desired values as the theorem states.

\section{Selection of the bus voltage value}

The direct application of the conditions given in the Theorem 1 could lead to the selection of a high value of the bus voltage. In order to set a practical (not too high) bus voltage value this work follows the standard design procedure provided by the equivalent control method [18].

The equivalent control is defined as the control value, $u_{e q}$, that confines the system exactly on the manifold $\sigma=0$ and it can be found assuming that $\dot{\boldsymbol{\sigma}}=0$. From (13) one gets:

$$
\dot{\boldsymbol{\sigma}}=\mathbf{f}_{\mathbf{2}}-v_{\text {bus }} \mathbf{u}_{e q}=0 \Rightarrow \mathbf{u}_{e q}=v_{b u s}^{-1} \mathbf{f}_{\mathbf{2}}
$$

where $\mathbf{u}_{e q}=\left[u_{a_{e q}}, u_{b_{e q}}, u_{c_{e q}}\right]^{T}$.

Notice that the function $\mathbf{f}_{2}$ in (21) particularizes (14) when $\dot{\sigma}=0$ and thus $i_{a}=i_{a}^{*}, i_{b}=i_{b}^{*}$ and $v_{n}=v_{n}^{*}$. Using the equivalent control method, the existence condition of the sliding mode can be guaranteed when $-1<u_{e q}<1$. Furthermore, the equivalent control vector given in (21) can be considered as the equivalent steady state duty cycles (with values $[-1,1])$ needed in the set of equations (1-3) to fulfil the requirement of perfect tracking of the current references.
In practice the value of $v_{b u s}$ is set from (20) preserving a maximum (minimum) value of the equivalent control lower (higher) than $1(-1)$ for the maximum (mimimum) value of $\mathbf{f}_{\mathbf{2}}$, and therefore ensures sliding mode for the expected nominal conditions of the machine, as the maximum angular speed or the maximum stator current. Of course, the selected value of $v_{\text {bus }}$ implies that the design condition of Theorem 1 could not be fulfilled in transients. In these cases, the control is saturated and the trajectories of the system will recover again the sliding motion.

Remark 4. The robustness of the method resides in that the control law in (15) does not depend on any system parameter, requiring only the references values $i_{a}^{*}, i_{b}^{*}, v_{n}^{*}$ and the measured values of $i_{a}, i_{b}, v_{n}$. The bound for $\mathbf{f}_{2}$, allows to select the value for $v_{\text {bus }}$ that guaranties the existence of the sliding mode. In other words, the measurement of $\mathbf{f}_{2}$ is not required by the proposed sliding mode controllers.

\section{Reference generator}

The current controller previously designed works on the $a b c$ reference frame and therefore the values $i_{a}^{*}, i_{b}^{*}$ and $v_{n}^{*}$ should be provided. In this work the proposed sliding mode current control has been tested when the motor speed or torque are regulated. In the first case a well-known IP speed controller [33] is used as an external controller to provide the torque reference value, which will be proportional to the $i_{q}^{*}$ since $i_{d}^{*}$ will be set to 0 . In the second case, the $i_{q}^{*}$ is provided directly in order to fix the motor torque keeping $i_{d}^{*}$ equal to zero. With regards to $v_{n}$, the default average reference value is set to 0 .

\section{SWITCHING FREQUENCY ANALYSIS OF THE PROPOSED CONTROL}

The control law designed in equation (15) assumes theoretically infinite switching frequency of the control action. Under this assumption, the system trajectories are confined to the manifold $\sigma=0$. From an implementation point of view, the switching frequency has to be bounded. The most common application of the sliding mode controller in practical systems is accomplished by means of hysteresis comparators instead of sign functions [25], [26]. In this case the control laws are rewritten as follows:

$$
u_{i}=\left\{\begin{array}{cll}
-1 & \text { if } & \sigma_{i}<-\Delta_{i}, \\
1 & \text { if } \quad & \sigma_{i}>\Delta_{i},
\end{array} \quad i \in\{a, b, c\}\right.
$$

and the trajectories are not confined to the manifold $\sigma=0$, but oscillate around it, within a boundary layer $2 \Delta>0$.

From the control law defined in (22), it is obvious that the equivalent control, which is a continuous variable dependent on unknown function $f_{2}$, cannot be attained. Therefore, the control will have a low frequency component, that can be understood as the equivalent control value, which will place the system on the desired surface, and a high frequency component oscillating around the surface [18]. Using the equivalent control definition one can rewrite (13) as:

$$
\dot{\boldsymbol{\sigma}}=v_{\text {bus }}\left[\mathbf{u}_{e q}-\mathbf{u}\right]
$$


From (23) the estimated switching period of the control signal can be obtained [34] when a comparator with a fixed hysteresis band, $\Delta$, as Figure 2 shows, is used. In accordance with figure 2 the switching period, $t_{s w}$, is given by:

$$
t_{s w}=t_{(u=1)}+t_{(u=-1)}=\frac{-2 \Delta}{\dot{\sigma}_{u=1}}+\frac{2 \Delta}{\dot{\sigma}_{u=-1}}
$$

Hence, using again the equivalent control method, the switching period for each control signal can be easily derived from (21), (23) and (24).

$$
t_{s w_{i}}=\frac{4 \Delta_{i} v_{b u s}}{v_{b u s}^{2}-f_{2_{i}}^{2}}
$$

where sub index $i \in\{a, b, c\}$ and $\left\{\Delta_{a}, \Delta_{b}, \Delta_{c}\right\}$ are the hysteresis bands of each surface. Equation 25 only makes sense when its denominator is higher than zero. Theorem 1 states that in order to ensure sliding motion, $v_{b u s}>\max \left\{f_{2_{a} M}\right.$, $\left.f_{2_{b} M}, f_{2_{c} M}\right\}+\eta$, being $\eta>0$. This implies a non null and positive denominator in (25). In cases where the sliding mode can not be guaranteed (during transients), the control action is saturated to one of its values ( -1 or 1$)$ until the system trajectories recover the conditions for the sliding mode to exist. During these conditions, where the control action does not switch, (25) does not apply.

All the parameters in (25) are constant except $f_{2_{i}}$. From the definition of $f_{2 i}$ stated previously, it can be realized that the switching periods vary with the motor parameters, stators currents, back-emf voltages and speed. From (25) arises a minimum switching period when $f_{2_{i}}=0$ and a maximum switching period when $f_{2_{i}}=f_{2_{i} M}$.

The switching period can be controlled by means of a variable hysteresis band. Similar approaches have been proposed by several authors for different power converters [35]. Notice how in order to properly adjust the hysteresis value, $\mathbf{f}_{2}$ must be known. The measure of vector $\mathbf{f}_{2}$ becomes impossible from a practical point of view, since it depends on unknown perturbing signals and motor parameters. Measure the equivalent controls constitute an alternative approach. Once the sliding motion is reached, according to [18], the equivalent control $\mathbf{u}_{e q}$ can be measured by low pass filtering the control signals $\mathbf{u}$. With the measured signals of the $\mathbf{u}_{e q}$ and with a known bus voltage, $\mathbf{f}_{\mathbf{2}}$ can be estimated using (21). With such estimation, it is possible to adjust the hysteresis band, according to (26), in order to get the desired switching period:

$$
\Delta_{i}=0.25 t_{s w_{i}}^{*} v_{b u s}\left(1-u_{i_{e q}}^{2}\right) .
$$

Remark 5. The switching period calculated in (24) and (25) assumes piecewise linear behavior of the switching surface. This means that, the surface slopes are treated as constants during the entire switching period. This assumption can be applied as long as the switching frequency is high enough with respect to the system dynamics.

Remark 6. Arbitrary values of hysteresis can lead the system far away from the sliding domain. As a consequence, an allowable range for the hysteresis has to be defined, within which the hysteresis band values have to "live", preserving

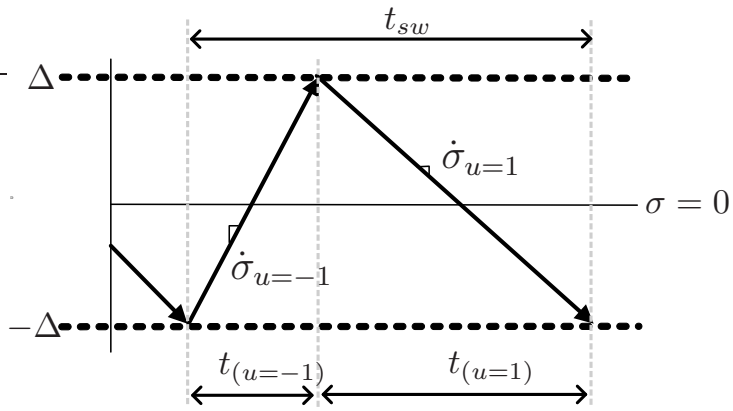

Fig. 2. Switching surface behavior within a constant amplitude boundary layer.

the sliding motion at any time and limiting the permissible switching periods to a safe levels as:

$$
\Delta_{i} \in\left[\Delta_{\min }, \Delta_{\max }\right] ; \Delta_{\min }, \Delta_{\max }>0
$$

where $\Delta_{\text {max }}$, has to be designed according to the minimum feasible value of the switching frequency, thus avoiding high current ripples and component saturation. Analogously, $\Delta_{\min }$ is related to the maximum switching frequency in order to do not overheat the power switches of the VSI.

\section{Simulations of Continuous time Sliding Mode CONTROL}

In this section some simulations are presented validating the sliding mode controllers designed in Section II and the performance of the switching periods derived in section III. The simulations have been developed using an accurate models created in MATLAB/SIMULINK and PSIM. The motor data and the VSI parameters are presented in Table I. The simulation results are shown in Figure 3.

The first graph shows the motor currents during this test, and the second one plots the speed, the torque and the reference torque which is varied from $8.1 \mathrm{Nm}$ to $-8.1 \mathrm{Nm}$. Under this condition, the electrical motor speed reaches a maximum value of $600 \mathrm{rad} / \mathrm{s}$, corresponding to a mechanical speed of 200 $\mathrm{rad} / \mathrm{s}$, see second plot. The third plot shows the time evolution of the switching period of $\sigma_{a}$, adjusting the hysteresis band according (26) in order to get a fixed switching period of $80 \mu \mathrm{s}$. Finally, the last graph shows the behaviour of the decoupled switching surface $\sigma_{a}$ (scaled $68 \cdot 10^{3}$ factor, see section VI for details) inside the hysteresis bands, where considering the further real time implementation. From the zoomed area on the right side it can be seen how the switching slopes are constant and the surface is correctly decoupled.

\section{Digital implementation of the Sliding Mode CONTROL}

As it has been introduced in the section III, when the piece-wise linear behaviour assumption holds, a sliding motion enforced in a hysteresis band comparator provides a known switching frequency. From the implementation point of view, the hysteresis comparator can be accurately built using analogue circuitry. However, the implementation in digital signal processors (DSP) degrades the performance due to the effects 

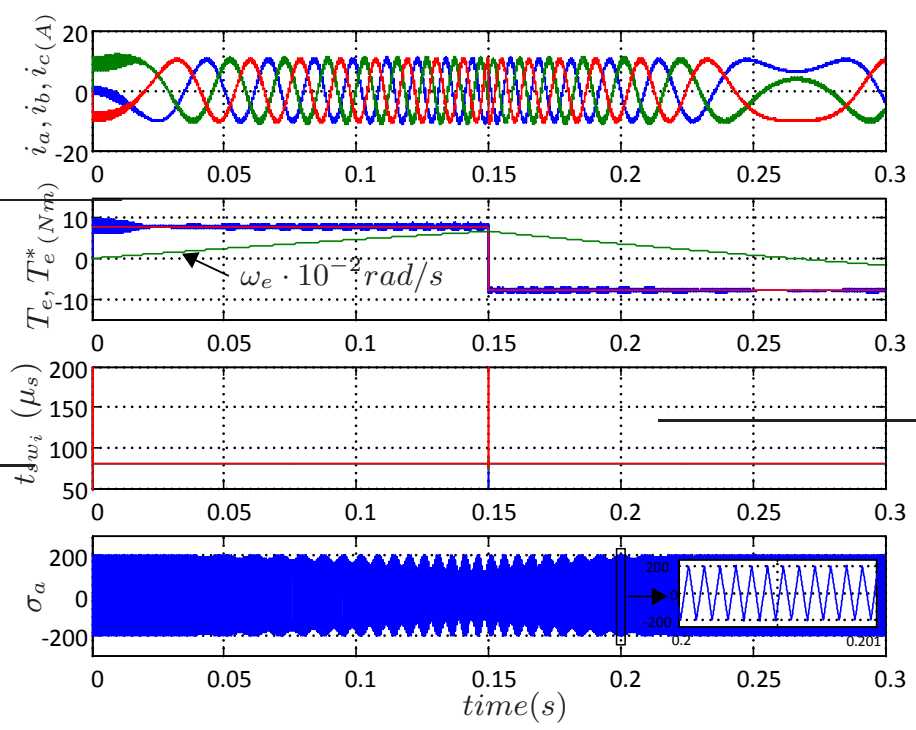

Fig. 3. Simulation results with a variable hysteresis band in continuous time system.

of the sampling period and computing delay. In order to properly implement the aforementioned controllers in a DSP, a predictive algorithm together with a specific PWM usage is introduced.

The procedure should be able to deliver the proper control action $u(t)$, in such a way the switching function $\sigma$ change its slopes sign just when it hits the hysteresis band $\Delta$. The desired performance is illustrated in figure 4 .

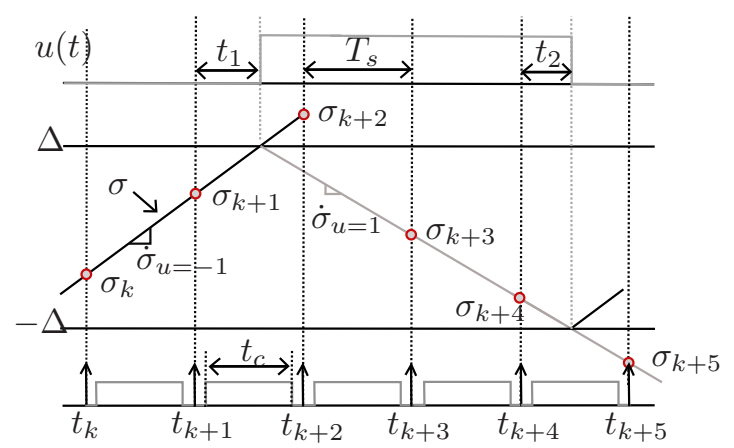

Fig. 4. Discretized switching surface desired behavior.

The microcontroller is able to perform actions only at the sampling time instants, $t_{k+1}, t_{k+2}, \ldots$ In order to make the switching action at time instant $t=t_{1}+t_{k+1}$, the following pulse width modulated control signal is applied:

$$
d=\left\{\begin{array}{cccc}
d_{k} & =0 & \text { at } & t=t_{k} \\
d_{k+1}=t_{1} / T_{s} & \text { at } & t=t_{k+1} \\
d_{k+2}=1 & \text { at } & t=t_{k+2}
\end{array}\right.
$$

According to Figure 4 the following duty ratio $d_{k+1}$, updated at $t=t_{k+1}$, makes possible the commutation at the desired time instant $t=t_{k+1}+t_{1}$ :

$$
d_{k+1}=\frac{\Delta-\sigma_{k+1}}{\sigma_{k+2}-\sigma_{k+1}} .
$$

Note that $d_{k+1}$ depends on the future sample $\sigma_{k+2}$. At this point, a predicted value of $\sigma_{k+2}$ is needed. Furthermore, a
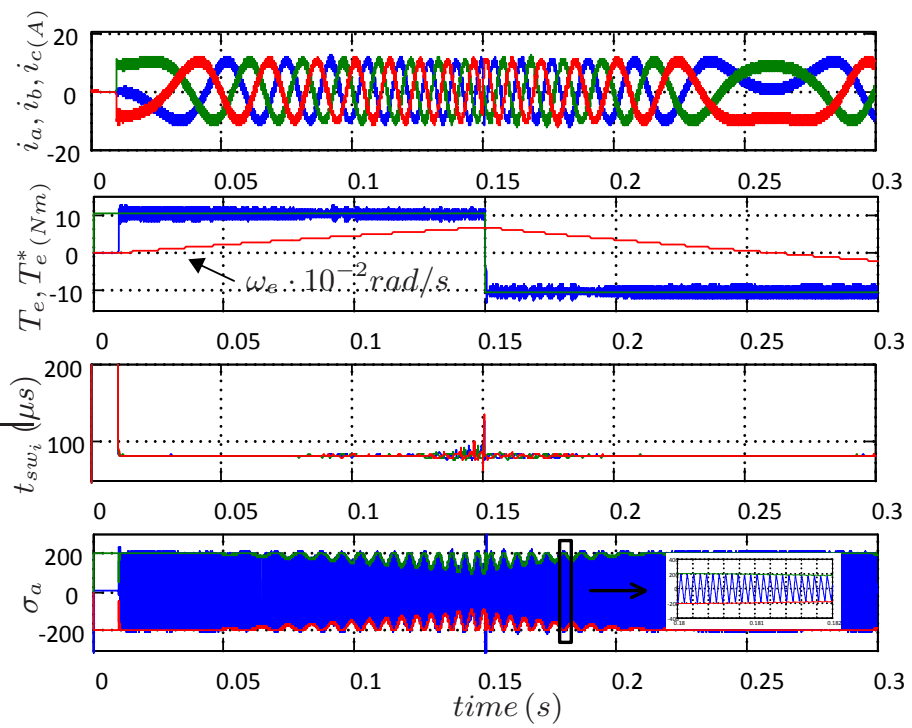

Fig. 5. Simulation results with a variable hysteresis band in a discretized simulation with the proposed prediction and PWM configuration.

sampling period delay should be taken into account, due to the computing time ( $t_{c}$ at figure 4$)$. This means that the duty cycle which can be applied at $t=t_{k+1}$, has to be calculated with the available information at $t=t_{k}$. Referred to (28) this implies a prediction of $\sigma_{k+1}$ and $\sigma_{k+2}$ at time instant $t=t_{k}$.

Therefore the control law is redefined, according to the predictions, at time instant $t=t_{k}$ :

$$
\left\{\begin{array}{lc}
\text { if } \hat{\sigma}_{k+2}>\Delta \text { then } & d_{k+1}=\frac{\Delta-\hat{\sigma}_{k+1}}{\hat{\sigma}_{k+2}-\hat{\sigma}_{k+1}} \\
\text { if } \hat{\sigma}_{k+2}<\Delta \text { then } & d_{k+1}=0
\end{array}\right.
$$

The prediction is performed according to (30) to each surface separately.

$$
\hat{\sigma}_{k+1}=\sigma_{k}+m_{k} T_{s} ; \quad \hat{\sigma}_{k+2}=\sigma_{k}+2 m_{k} T_{s}
$$

where $m_{k}$ is the time derivative of $\sigma$ at sample $k$. The prediction algorithm assumes that the surface time derivative remains constant between the samples, that is $m_{k}=m_{k+1}=$ $m_{k+2}$. From (23) the value of $m_{k}$ can be calculated from the measures of the equivalent control:

$$
m_{k}=\dot{\sigma}=v_{b u s}\left(u_{e q_{k}}-u(t)_{k}\right) .
$$

In order to validate the prediction algorithm and the PWM adjustment proposed in this work, the same simulation test presented at section IV has been done. The results are shown in Figure 5, when the sampling period is set to $T_{s}=5 \mu \mathrm{s}$. Notice that the desired performance, shown in figure 3 , is qualitatively preserved and the switching period is regulated to $80 \mu \mathrm{s}$.

Some inaccuracies are observed when the motor speed increases. The prediction of the surface will work fine only if the assumption $m_{k}=m_{k+1}=m_{k+2}$ is fulfilled. It should be noted that the surface time derivatives depend on the equivalent control (see (23)) and from (2), (14) and (21) it can be stated that when the speed motor rises, the surface slopes are not entirely constant along the switching period. It is also obvious that increasing the sampling frequency will improve the accuracy of the switching frequency regulation. 


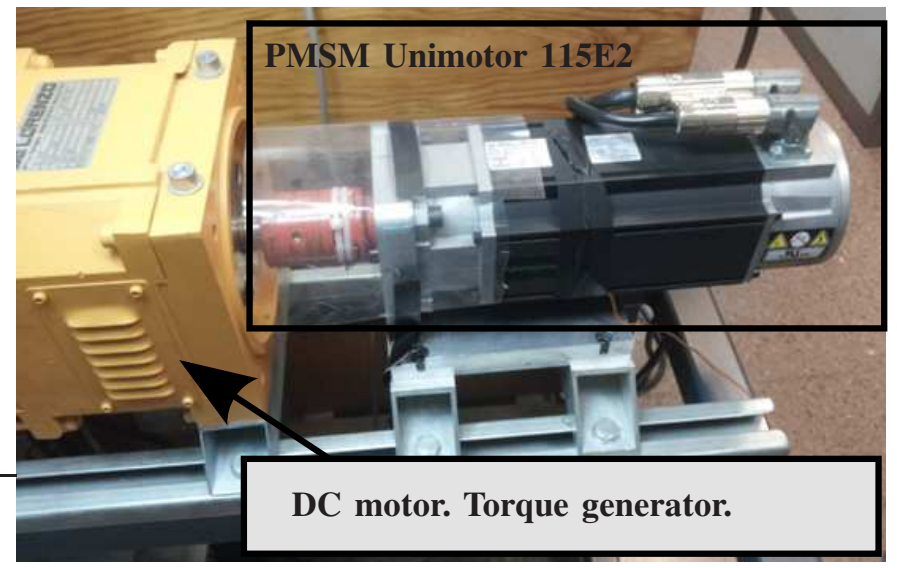

Fig. 6. Motor platform used in the experimental evaluation.

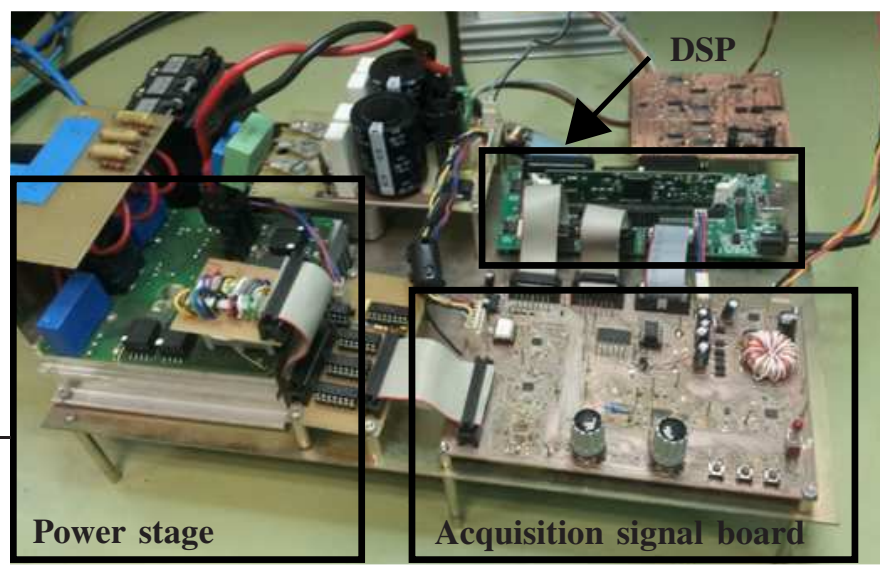

Fig. 7. Control boards.

\section{EXPERIMENTAL RESULtS}

The sliding mode control and the discrete-time implementation procedure proposed in the previous sections are tested experimentally at this stage. For this purpose, a platform based on a 3-phase PMSM (Unimotor 115E2 [36]) together with a VSI has been built in the laboratory, whose parameters are detailed in table I. Figures 6-7 show the experimental platform and the control stage, respectively. The machine type corresponds to an AC sinusoidal brushless servomotor. Among the different PMSMs, it can be classified as an SMPMSM (Surface Mount Permanent Magnet Synchronous Motor) which produces low, but non-negligible dependence of the stator inductances, $L_{a, b, c}$, as a function of the rotor position, which implies a good challenge for the SMC from the robustness point of view. Table I shows these values, which have been measured empirically using the high frequency current ripple flowing through the stators windings. The VSI power switches are the Infineon FS50R06W1E3. The motor currents $\left(i_{a}, i_{b}\right)$ are measured with the sensor LEM LAH 50-P and the $v_{\text {bus }}$ is acquired using a voltage divider through an isolated amplifier (ACPL-790A). Finally, the rotor position is provided by an encoder included in the motor shaft.

The control requires the measurement of the equivalent controls $\left(u_{a_{e q}}, u_{b_{e q}}, u_{c_{e q}}\right)$ for the variable hysteresis band
TABLE I

MOTOR DRIVE PARAMETERS

\begin{tabular}{lcc}
\hline \hline Parameter & Symbol & Value \\
\hline Power, Torque, Speed & $P, T_{e}, w_{e}$ & $2.54 \mathrm{~kW}, 8.1 \mathrm{~N} \cdot \mathrm{m}, 3000 \mathrm{rpm}$ \\
Motor Constants & $K_{T}, K_{e}$ & $0.93 \mathrm{Nm} / \mathrm{A}, 57 \mathrm{~V} / \mathrm{krpm}$ \\
Pole pairs & $p$ & 3 \\
Stator Inductances & $L_{a}, L_{b}, L_{c}$ & $1.5 \mathrm{mH} \pm 0.25 \mathrm{mH}$ \\
Stator Resistance & $R$ & $0.36 \Omega$ \\
Moment of Inertia & $J$ & $4.57 \cdot 10^{-3} \mathrm{~kg} \cdot \mathrm{m}^{2}$ \\
Friction Coefficient & $B$ & $8.75 \cdot 10^{-3} \mathrm{~N} \cdot \mathrm{m} \cdot \mathrm{s}$ \\
Bus Voltage & $\pm v_{b u s}$ & $\pm 175 \mathrm{~V}$ \\
\hline \hline & $\mathrm{IP}$ Speed controller \\
\hline Sampling Period & $T_{z w}$ & $5 \mathrm{~ms}$ \\
Settling time at $2 \%$ & $S T_{2}$ & 0.707 \\
Damping factor & $\zeta$ & \multicolumn{1}{c}{$\zeta$} \\
\hline \multicolumn{2}{c}{$k_{i}=\left(4.22^{2} \cdot J\right) /\left(\zeta^{2} \cdot S T_{2}^{2}\right) ; k_{p}=\left((2 \cdot 4.22 \cdot J) /\left(S T_{2}^{2}\right)\right)-B$} \\
\hline \hline
\end{tabular}

calculation and for the prediction algorithm, according to (26) and (31), respectively. In order to obtain an accurate measurements the IC LT2645-8 from Linear Technology is employed. This device receives the three PWM control signals $\left(u_{a}, u_{b}, u_{c}\right)$ and delivers their duty cycles, which correspond to the equivalent controls [18], [37]. Using the LT2645-8 instead of a low pass filter the problems related to the phase shift are overcome.

The proposed control algorithms are implemented with the microcontroller F28335 form Texas Instruments. The microcontroller acquires through the ADC peripheral the required signals $\left(i_{a}, i_{b}, v_{b u s}\right)$ and computes the switching surfaces defined in (8). Since $v_{n}$ voltage is not accessible, equation (5) is used instead where equal inductance values are assumed.

Once the microcontroller calculates the surface vector $\mathbf{S}$, the decoupled surface vector $\sigma$ is obtained applying (10) and (12) (assuming equal inductances). The control laws $\left(u_{a}, u_{b}, u_{c}\right)$ are computed using (29). For their evaluation, the predicted samples of $\sigma$, and the hysteresis band $\Delta_{i}$ are needed. The predicted samples are performed employing (30) and (31) and the variable hysteresis band is calculated according to (26).

The microcontroller executes all the aforementioned tasks with a sampling period of $T_{s}=5 \mu \mathrm{s}$, except for the evaluation of the variable hysteresis band which is updated at $T_{s}=125 \mu \mathrm{s}$. In order to accelerate the computing time in the real execution, equations $(8,10,29,30,31)$ are computed using fixed point operations, which are twice faster than floating point operation in the F28335. As a consequence, the

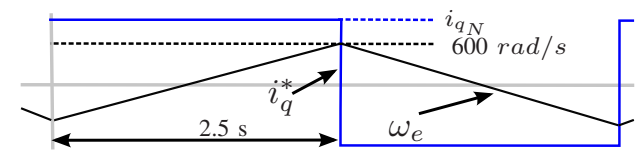

Fig. 8. $i_{q}^{*}$ applied during the test and the resulting profile of the motor mechanical speed. 


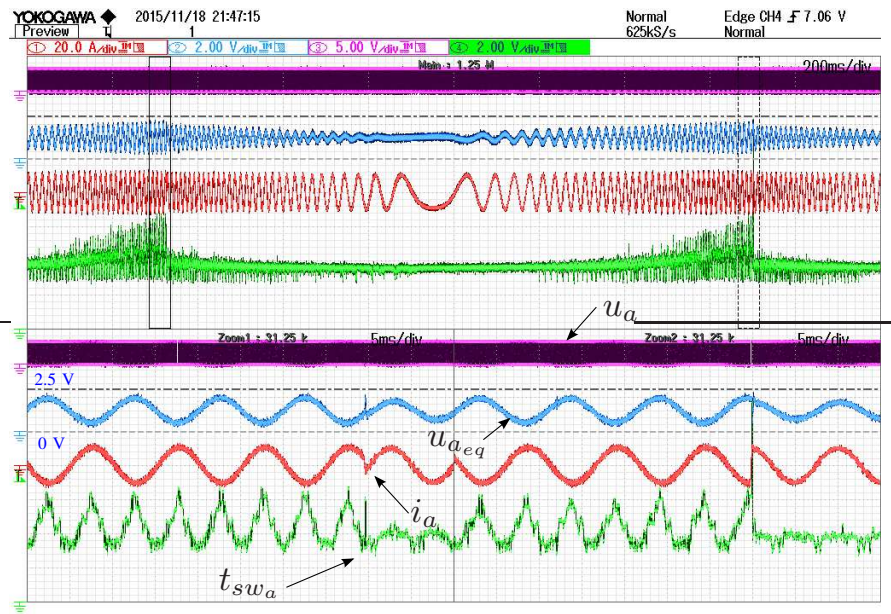

Fig. 9. Experimental results of a torque reversal control with a fixed hysteresis band.

switching surfaces $\sigma_{i}$ are scaled by a factor of $68 \cdot 10^{3}$ in order to avoid numerical inaccuracies.

The experimental tests consist in a torque reversal control and in a speed control. On one hand, the torque reversal control allows us to validate the expected fast transient response of the sliding mode current control designed in section II, the discrete-time control implementation procedure presented in section $\mathrm{V}$ and the different system responses when a fixed or a variable hysteresis bands are used. On the other hand, the speed control lets us to observe the variations of the three switching periods when fixed and variable hysteresis bands at constant speed are applied. When required, the speed control was performed by an IP controller tuned with the pole placement technique and whose specifications are detailed on Table I.

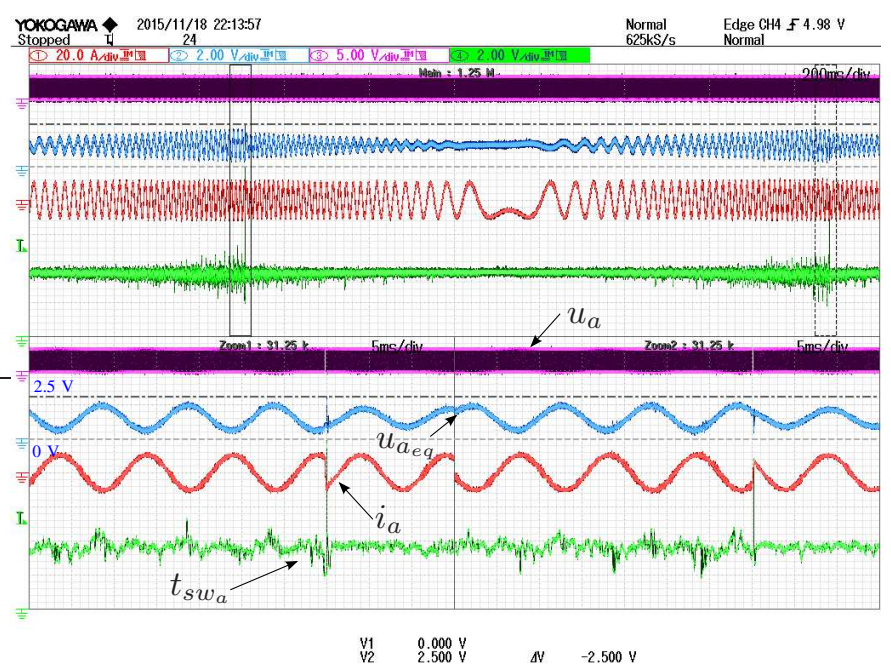

Fig. 10. Experimental results of torque reversal control with a variable hysteresis band.

\section{A. Torque Reversal Control}

The references $i_{d}^{*}$ and $v_{n}^{*}$ are fixed both to 0 , being $i_{q}^{*}$ proportional to the reference torque. The applied reference

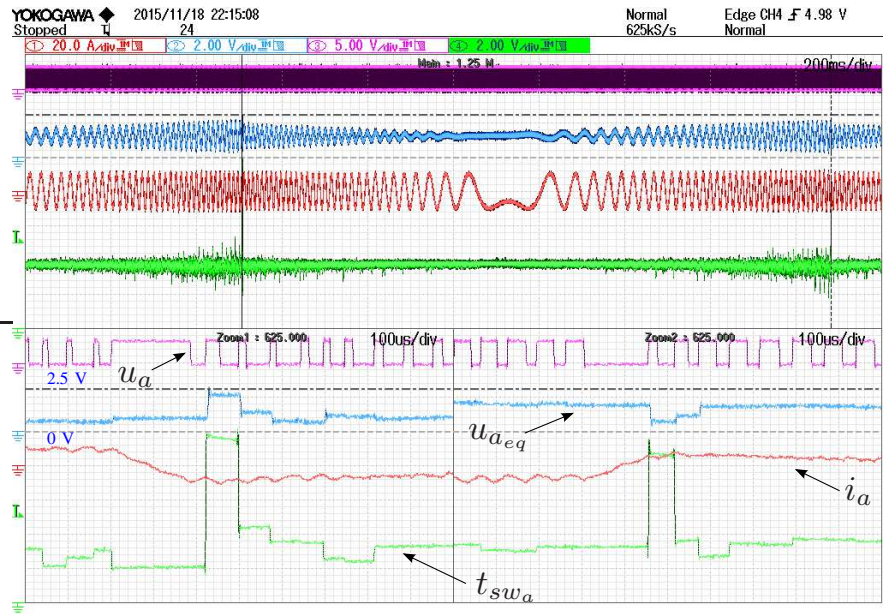

Fig. 11. Experimental results of torque reversal control with a variable hysteresis band. Transient detail.

torque profile is shown in Fig. 8, together with behaviour of the mechanical motor speed at these conditions.

Figures 9, 10 and 11 show the following signals: the equivalent control ( $u_{a_{e q}}$, blue), the control action ( $u_{a}$, magenta), the switching period $\left(t_{s w_{a}}\right.$, green) and phase current $\left(i_{a}\right.$, red $)$ of the phase $a$.

The equivalent control is scaled such that $2.5 \mathrm{~V}$ and $0 \mathrm{~V}$ correspond to 1 and -1 , respectively. In the same way, the control action presented in the graphs evolves from 0 to $3.3 \mathrm{~V}$, where $3.3 \mathrm{~V}$ correspond to $u_{a}=1$ and $0 \mathrm{~V}$ to $u_{a}=-1$. An analogue circuit has been designed for measuring the switching periods $t_{s w_{a}}, t_{s w_{b}}, t_{s w_{c}}$. The sensibility of the circuit is of $0.5 \mathrm{~V} / \mu \mathrm{s}$.

From Figures 9, 10 and 11 the expected good performance of the proposed sliding controller is confirmed in all the cases. Figure 9 shows the experimental results for fixed hysteresis band of $\Delta_{i}=224$. The amplitude of the measured equivalent control (blue signal in the zoomed area) is of $(2 \mathrm{~V}-0.5 \mathrm{~V}) / 2.5=0.6$. Therefore, $f_{2_{i}}$ becomes $175 \mathrm{~V} \cdot 0.6=$ $105 \mathrm{~V}$. Then, recalling that the switching surface $\sigma_{a}$ has been scaled by $68 \cdot 10^{3},(25)$ yields a theoretical values of $75 \mu \mathrm{s}$ as minimum switching period and a maximum of $118 \mu \mathrm{s}$. The measured switching period (green signal in the plot) is $72 \mu \mathrm{s}$ as minimum value and $120 \mu \mathrm{s}$ as maximum one. These results validate the prediction and the PWM adjustment algorithms, since the expected and the real switching periods are close. The system non-idealities such as the VSI dead time or the motor saliency are not considered in (25), justifying the small differences between them. Figure 10 shows the experimental results when the hysteresis band is adjusted according to (26) with a desired switching period of $t_{s w}^{*}=80 \mu \mathrm{s}$. Comparing Figures 9 and 10 the performance improvement becomes evident and the oscillation of the switching period is considerably reduced. The zoomed areas in Figure 11 show the details of the current transient of $i_{a}$ (red signal in the graph) when $i_{q}^{*}$ changes from $10 \mathrm{~A}$ to $-10 \mathrm{~A}$ and vice versa. Notice the brief transient responses, with a settling time less than $200 \mu \mathrm{s}$.

Finally, Figure 12 shows the data stored by the DSP during the previous test and downloaded after execution, for the 


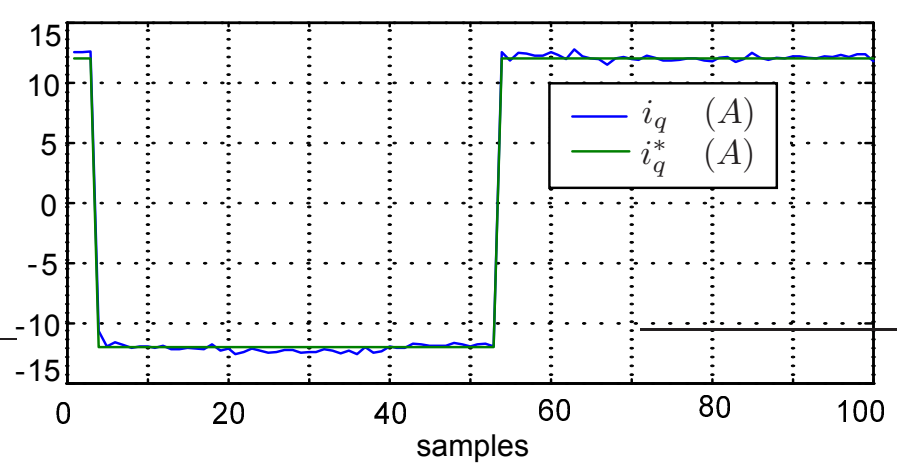

Fig. 12. Torque reversal experimental test, from -12 to $12 \mathrm{Nm}$. Data downloaded from the DSP after execution. Sampling of $25 \mathrm{~ms}$.

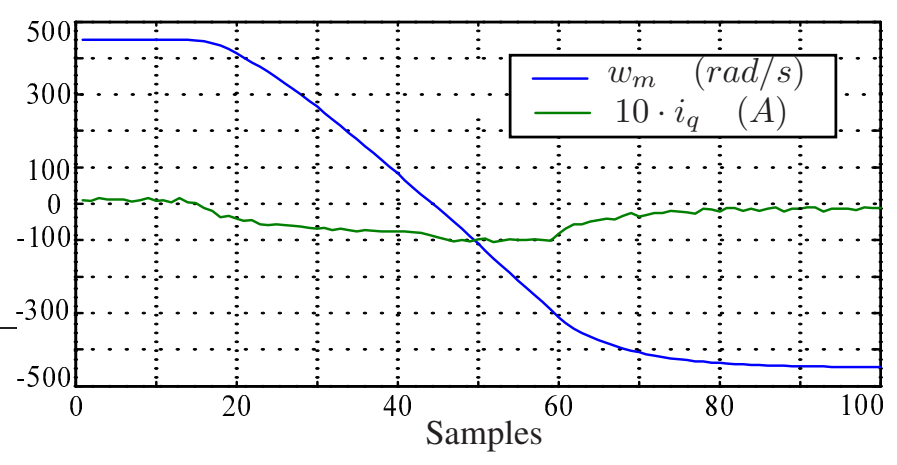

Fig. 13. Speed reversal experimental test, from -450 to $450 \mathrm{rad} / \mathrm{s}$. Data downloaded from the DSP after execution. Sampling of $25 \mathrm{~ms}$.

variable hysteresis case, of $i_{q}, i_{q}^{*}$.

\section{B. Speed Control}

An IP speed control [33] is applied to the PMSM, which is validated through a speed reversal test. The results of this test (provided by the DSP's data stored) are depicted in Figure 13. In order to stand out the effect of use variable or fixed hysteresis band values, the motor speed is regulated at 600 $\mathrm{rad} / \mathrm{s}$, being the values of $\Delta$ suddenly varied from a fixed value to a variables ones, according to (26) for a desired switching period of $t_{s w}^{*}=80 \mu s$.

Figure 14 shows the three switching periods of the decoupled surfaces $\sigma_{a}, \sigma_{b}, \sigma_{c}$ (blue, magenta and green signals) together with $i_{a}$ (red signal). The behavior of the three switching periods and the proper rejection of the switching period oscillation when the variable hysteresis is employed confirm the overall good performance of the proposed controller.

\section{CONCLUSIONS AND FUTURE RESEARCH}

An SMC for PMSMs has been designed combining successfully the advantages of the traditional FOC and DTC, which are constant switching frequency and high dynamics, respectively. Besides, all the SMC benefits such as robustness and system order reduction are preserved in a digitally implemented system.

A decoupling algorithm has been designed, not only to allow the proper implementation of the SMC delivering the control laws directly to the inverter but also to impose constant

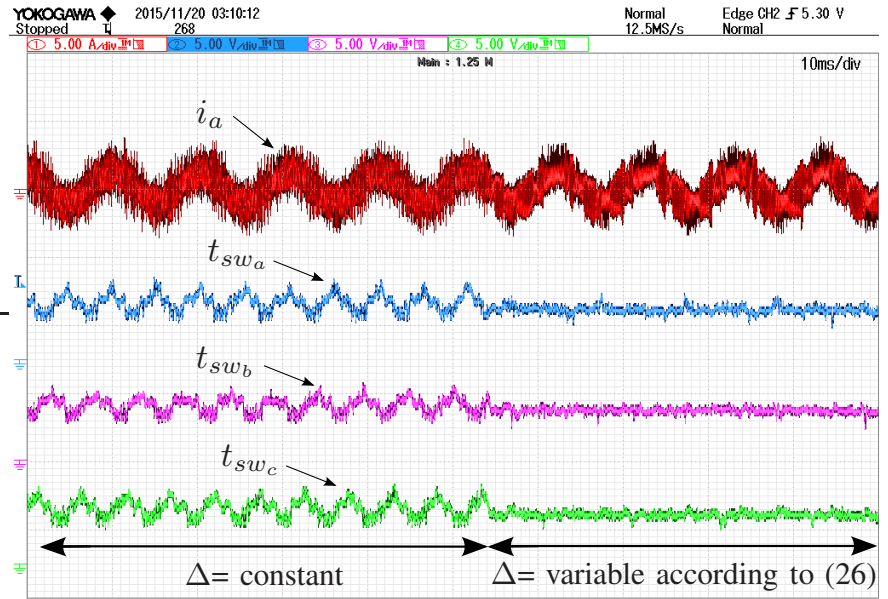

Fig. 14. Switching period of each decoupled surfaces rotating at 600 $\mathrm{rad} / \mathrm{s}$.

slopes in the sliding surfaces and consequently bringing the possibility to further regulate the switching frequency. Hence, a variable hysteresis band calculation that keeps the switching period constant, conceived in continuous time domain, has been designed.

Trough a detailed methodology, the designed controllers have been successfully implemented using a DSP, as it could be inferred from the presented experimental results under different conditions in an off-the-shelf PMSM.

Once the control proposal has been presented and successfully evaluated, a deep comparison among the control structures of FOC, DTC and SMC, in order to discern the benefits and drawback of each one, constitutes a further research subject.

\section{REFERENCES}

[1] P. C. Krause, O. Wasynczuk, S. D. Sudhoff, and S. Pekarek, Analysis of electric machinery and drive systems, vol. 75. John Wiley \& Sons, 2013.

[2] X. Liu, H. Chen, J. Zhao, and A. Belahcen, "Research on the performances and parameters of interior PMSM used for electric vehicles," IEEE Transactions on Industrial Electronics, vol. 63, no. 6, pp. 35333545, Jun. 2016.

[3] S. K. Kommuri, M. Defoort, H. R. Karimi, and K. C. Veluvolu, "A robust observer-based sensor fault-tolerant control for PMSM in electric vehicles," IEEE Transactions on Industrial Electronics, vol. 63, no. 12, pp. 7671-7681, Dec. 2016.

[4] M. Haque, M. Negnevitsky, and K. Muttaqi, "A novel control strategy for a variable-speed wind turbine with a permanent-magnet synchronous generator," IEEE Transactions on Industry Applications, vol. 46, no. 1, pp. 331-339, Jan. 2010.

[5] F. Blaschke, "The principle of field orientation as applied to the new transvector closed-loop control system for rotating field machines," 1972.

[6] I. Takahashi and T. Noguchi, "A new quick-response and high-efficiency control strategy of an induction motor," IEEE Transactions on Industry Applications, vol. IA-22, no. 5, pp. 820-827, Sep. 1986.

[7] U. Baader, M. Depenbrock, and G. Gierse, "Direct self control of inverter-fed induction machine, a basis for speed control without speedmeasurement," in Annual Meeting of the IEEE Industry Applications Society, pp. 486-492 vol.1, Oct. 1989.

[8] L. Zhong, M. F. Rahman, W. Y. Hu, and K. Lim, "Analysis of direct torque control in permanent magnet synchronous motor drives," IEEE Transactions on Power Electronics, vol. 12, no. 3, pp. 528-536, 1997. 
[9] M. F. Rahman, M. E. Haque, L. Tang, and L. Zhong, "Problems associated with the direct torque control of an interior permanent-magnet synchronous motor drive and their remedies," IEEE Transactions on Industrial Electronics, vol. 51, no. 4, pp. 799-809, 2004.

[10] Y.-R. Mohamed, "Design and implementation of a robust current-control scheme for a PMSM vector drive with a simple adaptive disturbance observer," IEEE Transactions on Industrial Electronics, vol. 54, no. 4, pp. 1981-1988, Aug. 2007.

[11] Y. Zhang, J. Zhu, W. Xu, and Y. Guo, "A simple method to reduce torque ripple in direct torque-controlled permanent-magnet synchronous motor by using vectors with variable amplitude and angle," IEEE Transactions on Industrial Electronics, vol. 58, no. 7, pp. 2848-2859, 2011.

[12] Y. Zhang and J. Zhu, "Direct torque control of permanent magnet synchronous motor with reduced torque ripple and commutation frequency," IEEE Transactions on Power Electronics, vol. 26, no. 1, pp. 235-248, 2011

[13] A. Mora, A. Orellana, J. Juliet, and R. Cárdenas, "Model predictive torque control for torque ripple compensation in variable-speed PMSMs," IEEE Transactions on Industrial Electronics, vol. 63, no. 7, pp. 4584-4592, Jul. 2016.

[14] M. Vafaie, B. Mirzaeian Dehkordi, P. Moallem, and A. Kiyoumarsi, "A new predictive direct torque control method for improving both steadystate and transient-state operations of the PMSM," IEEE Transactions on Power Electronics, vol. 31, no. 5, pp. 3738-3753, May. 2016.

[15] C. Ortega, A. Arias, C. Caruana, J. Balcells, and G. M. Asher, "Improved waveform quality in the direct torque control of matrix-converter-fed PMSM drives," IEEE Transactions on Industrial Electronics, vol. 57, no. 6, pp. 2101-2110, 2010

[16] Y. Ren, Z. Zhu, and J. Liu, "Direct torque control of permanent-magnet synchronous machine drives with a simple duty ratio regulator," IEEE Transactions on Industrial Electronics, vol. 61, no. 10, pp. 5249-5258, 2014.

[17] O. Sandre-Hernandez, R. Morales-Caporal, J. Rangel-Magdaleno, H. Peregrina-Barreto, and J. Hernandez-Perez, "Parameter identification of PMSMs using experimental measurements and a PSO algorithm," IEEE Transactions on Instrumentation and Measurement, vol. 64, no. 8, pp. 2146-2154, Aug. 2015.

[18] V. Utkin, J. Guldner, and J. Shi, Sliding mode control in electromechanical systems, vol. 34. CRC press, 2009.

[19] Y. Fan, L. Zhang, M. Cheng, and K. Chau, "Sensorless SVPWM-FADTC of a new flux-modulated permanent-magnet wheel motor based on a wide-speed sliding mode observer," IEEE Transactions on Industrial Electronics, vol. 62, no. 5, pp. 3143-3151, 2015.

[20] H. Kim, J. Son, and J. Lee, "A high-speed sliding-mode observer for the sensorless speed control of a PMSM," IEEE Transactions on Industrial Electronics, vol. 58, no. 9, pp. 4069-4077, Sep. 2011.

[21] M. L. Corradini, G. Ippoliti, S. Longhi, and G. Orlando, "A quasisliding mode approach for robust control and speed estimation of PM synchronous motors," IEEE Transactions on Industrial Electronics, vol. 59, no. 2, pp. 1096-1104, 2012.

[22] J. Rivera Dominguez, A. Navarrete, M. A. Meza, A. G. Loukianov, and J. Canedo, "Digital sliding-mode sensorless control for surface-mounted PMSM," IEEE Transactions on Industrial Informatics, vol. 10, no. 1, pp. 137-151, 2014.

[23] W. Gao, Y. Wang, and A. Homaifa, "Discrete-time variable structure control systems," IEEE Transactions on Industrial Electronics, , vol. 42, no. 2, pp. 117-122, 1995 .

[24] K. Jezernik, J. Korelic, and R. Horvat, "PMSM sliding mode FPGAbased control for torque ripple reduction," IEEE Transactions on Power Electronics, vol. 28, no. 7, pp. 3549-3556, 2013.

[25] L. Malesani and P. Tenti, "Performance optimization of Cuk converters by sliding-mode control," IEEE Transactions on Power Electronics, vol. 10, no. 3, pp. 302-309, 1995.

[26] S.-P. Huang, H.-Q. Xu, and Y.-F. Liu, "Sliding-mode controlled Cuk switching regulator with fast response and first-order dynamic characteristic," in Power Electronics Specialists Conference, 1989. PESC'89 Record., 20th Annual IEEE, pp. 124-129. IEEE, 1989.

[27] A. Sabanovic and D. B. Izosimov, "Application of sliding modes to induction motor control," IEEE transactions on industry applications, vol. 1, no. IA-17, pp. 41-49, 1981.

[28] P. Cortés, M. P. Kazmierkowski, R. M. Kennel, D. E. Quevedo, and J. Rodríguez, "Predictive control in power electronics and drives," IEEE Transactions on Industrial Electronics, vol. 55, no. 12, pp. 4312-4324, 2008

[29] C. Carrejo, E. Vidal-Idiarte, R. Giral, and L. Martinez-Salamero, "Predictive digital interpolation current control for dc-dc power converters," IET Power Electronics, vol. 2, no. 5, pp. 545-554, 2009.
[30] M. Vafaie, B. Mirzaeian Dehkordi, P. Moallem, and A. Kiyoumarsi, "A new predictive direct torque control method for improving both steadystate and transient-state operations of the pmsm," IEEE Trans. Power Electron., vol. 31, no. 5, pp. 3738-3753, May. 2016.

[31] H. Zhu, X. Xiao, and Y. Li, "Torque ripple reduction of the torque predictive control scheme for permanent-magnet synchronous motors," IEEE Transactions on Industrial Electronics, vol. 59, no. 2, pp. 871-877, 2012.

[32] W. Yan, J. Hu, V. Utkin, and L. Xu, "Sliding mode pulsewidth modulation," IEEE transactions on power electronics, vol. 23, no. 2, pp. 619-626, 2008

[33] K.-T. Chang, T.-S. Low, and T.-H. Lee, "An optimal speed controller for permanent-magnet synchronous motor drives," IEEE Transactions on Industrial Electronics, vol. 41, no. 5, pp. 503-510, Oct. 1994.

[34] H. Buhler, "Regulation using sliding mode," Lausanne Switzerland: Presses Polytechniques Romandes, 1985.

[35] J. Ruiz, S. Lorenzo, I. Lobo, and J. Amigo, "Minimal UPS structure with sliding mode control and adaptive hysteresis band," in 16th Annual Conference of IEEE Industrial Electronics Society, IECON, pp. 10631067, 1990.

[36] PMSM

Manual"http://www.emersonindustrial.com/ en-en/documentcenter/ControlTechniques/Brochures/ unimotor_fm_product_data.pdf” accessed 29-December-2015.

[37] H. Sira-Ramirez, "Differential geometric methods in variable-structure control," International Journal of Control, vol. 48, no. 4, pp. 1359-1390, 1988 .

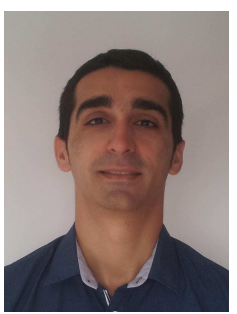

Víctor Repecho received the B.S and M.S. degrees in electronic engineering from the Universitat Politècnica de Catalunya (UPC), Barcelona, Spain, in 2006 and 2012, respectively. Since 2010, he has been a development Engineer with the Institute of Industrial and Control Engineering (IOC), UPC. His research fields are related to digital control, nonlinear control, and control of power electronic converters.

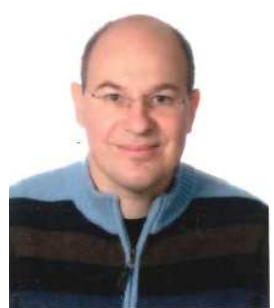

Domingo Biel received the B.S, M.S. and $\mathrm{Ph}$.D. degrees in telecommunications engineering from the Universitat Politècnica de Catalunya (UPC), Barcelona, Spain, in 1990, 1994 and 1999, respectively. Since 1998, he has been an Associate Professor in the Electronic Engineering Department, UPC, where he teaches power electronics and control theory. He is the coauthor of around 20 papers in international journals and more than 50 communications in international conferences. His research fields are related to nonlinear control and its application to renewable energy systems and power electronics.

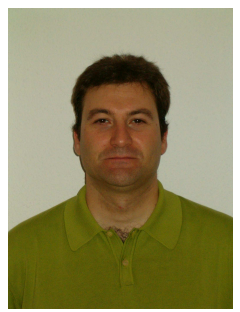

Antoni Arias received the BEng degree in electrical engineering, MEng and PhD degrees in control and electronic engineering from the Universitat Politècnica de Catalunya (UPC), Catalonia, Spain, in 1993, 1997 and 2001 respectively. From 1992/95 he worked at a local industrial electronics company. Since 1996 he has been a Lecturer at the UPC and was appointed as an Associate Professor in 2002. In 2003/04 he joined as a Visiting Fellow the PEMC Group at the University of Nottingham, UK. In 2011/12 he was a MCF invité at the GeePs, France. His research interests include sensorless variable-speed drive systems, power electronics converters and control strategies. 